\title{
Nuclear p53-mediated repression of autophagy involves PINK1 transcriptional down-regulation
}

\author{
Thomas Goiran $^{1} \cdot$ Eric Duplan $\unrhd^{1} \cdot$ Lila Rouland $^{1} \cdot$ Wejdane el Manaa ${ }^{1} \cdot$ Inger Lauritzen ${ }^{1} \cdot$ Julie Dunys ${ }^{1} \cdot$ Han You $^{2}$. \\ Frédéric Checler ${ }^{1} \cdot$ Cristine Alves da Costa $^{1}$
}

Received: 13 February 2017 / Revised: 29 September 2017 / Accepted: 18 October 2017

(c) ADMC Associazione Differenziamento e Morte Cellulare 2018

\begin{abstract}
p53 is a transcription factor that is implicated in the control of both apoptotic and autophagic cell death. This tumor suppressor elicits both pro-autophagic and anti-autophagic phenotypes depending of its intracellular localization. The ability of p53 to repress autophagy has been exclusively associated to its cytoplasmic localization. Here, we show that transcriptional activity of p53 also contributes to autophagy down-regulation. Thus, nuclear p53 controls PINK1, a key protein involved in the control of mitophagy, by repressing its promoter activity, protein and mRNA levels, ex-vivo and in vivo. We establish that deletion of an identified p53 responsive element on PINK1 promoter impacts p53-mediated PINK1 transcriptional repression and we demonstrate a p53-PINK1 physical interaction by chromatin immunoprecipitation. Accordingly, we show that only nuclear p53 accounts for its ability to repress PINK1 gene transcription. Further, we demonstrate ex-vivo and in vivo that p53 invalidation in human cells increases LC3 maturation as well as optineurin and NDP52 autophagy receptors expression and down-regulates TIM23, TOM20 and HSP60 mitophagy markers. Importantly, this phenotype is mimicked by TP53 invalidation in mice brain. Finally, by combining pharmacological and genetic approaches, we show that the p53-mediated negative regulation of autophagy is PINK1-dependent. Thus pifithrin- $\alpha$ mediated blockade of p53 transcriptional activity enhances LC3 maturation and reduces p62, TIM23, TOM20 and HSP60 protein levels. This pifithrin- $\alpha$-associated pro-mitophagy phenotype is fully abolished by PINK1 depletion. This data unravels a novel pathway by which nuclear p53 can repress autophagy/mitophagy that could underlie important dysfunctions in both neurodegenerative and cancer diseases.
\end{abstract}

\section{Introduction}

p53 is a key multifunctional protein, the implication of which has been extensively investigated in various pathologies. It is a transcription factor that may positively or

Edited by G. Del Sal

Electronic supplementary material The online version of this article (https://doi.org/10.1038/s41418-017-0016-0) contains supplementary material, which is available to authorized users.

Cristine Alves da Costa

acosta@ipmc.cnrs.fr

1 Université Côte d'Azur, INSERM, CNRS, IPMC, team labeled "Laboratory of Excellence (LABEX) Distalz", 660 route des Lucioles, 06560 Sophia-Antipolis, Valbonne, France

2 State Key Laboratory of Cellular Stress Biology, Innovation Center for Cell Signaling network, School of Life Sciences, Xiamen University, Xiamen, Fujian 361102, China negatively regulate a huge number of genes [1] involved in cell-fate control mechanisms linked to both apoptosis and autophagy. There is a consensus concerning the fact that p53-dependent control of autophagy is directly driven by its subcellular localization [2]. Thus, in stress conditions, p53 acts as a pro-autophagic mediator [2, 3] by modulating genes implicated in the regulation of mTOR (mammalian Target Of Rapamycine), a negative modulator of autophagy [4]. p53 also controls the transcription of Death-Associated Protein Kinase-1 [5] and Damage-Regulated Autophagy Modulator [6], two key players of autophagic response. In contrast to this transcriptional factor mediated proautophagic phenotype, cytoplasmic p53 can repress autophagy in absence of any cellular stress [7,8]. This inhibitory effect is independent of its transcriptional function and molecularly linked to the activation of the AMPKdependent inhibition of mTOR signaling cascade [7].

Interestingly, several works have shown a functional interplay between p53 and parkin. Thus, in physiological 
Fig. 1 Pharmacological modulation of $\mathrm{p} 53$ impacts PINK1 transcription. SH-SY5Y cells were treated with vehicle (CT), etoposide (ETO, $150 \mu \mathrm{M}$, $16 \mathrm{~h}$ ) or pifithrin-alpha (PFT, 10 $\mu \mathrm{M}, 16 \mathrm{~h}$ ) then PINK1 protein $(\mathbf{a}, \mathbf{d}, N=9)$, promoter activity (b, $N=9)$ and mRNA levels $(\mathbf{c}, \mathbf{e}, N=6)$ were analyzed as described in the Methods section. p53 and actin immunoreactivities are provided as read-out of p53 activation and gel loading controls $(\mathbf{a}, \mathbf{d}$, respectively). Bars represent the means \pm SEM of 3 independent experiments performed in triplicate $(\mathbf{a}, \mathbf{b}, \mathbf{d})$ or duplicates $(\mathbf{c}, \mathbf{e})$ and are expressed as percentage of vehicle-treated control cells. Statistical analyses were performed with

GraphPad Prism software by using unpaired Student's t-test. Significant differences are: $* p<$ $0.05, * * p<0.01, * * * p<0.001$.
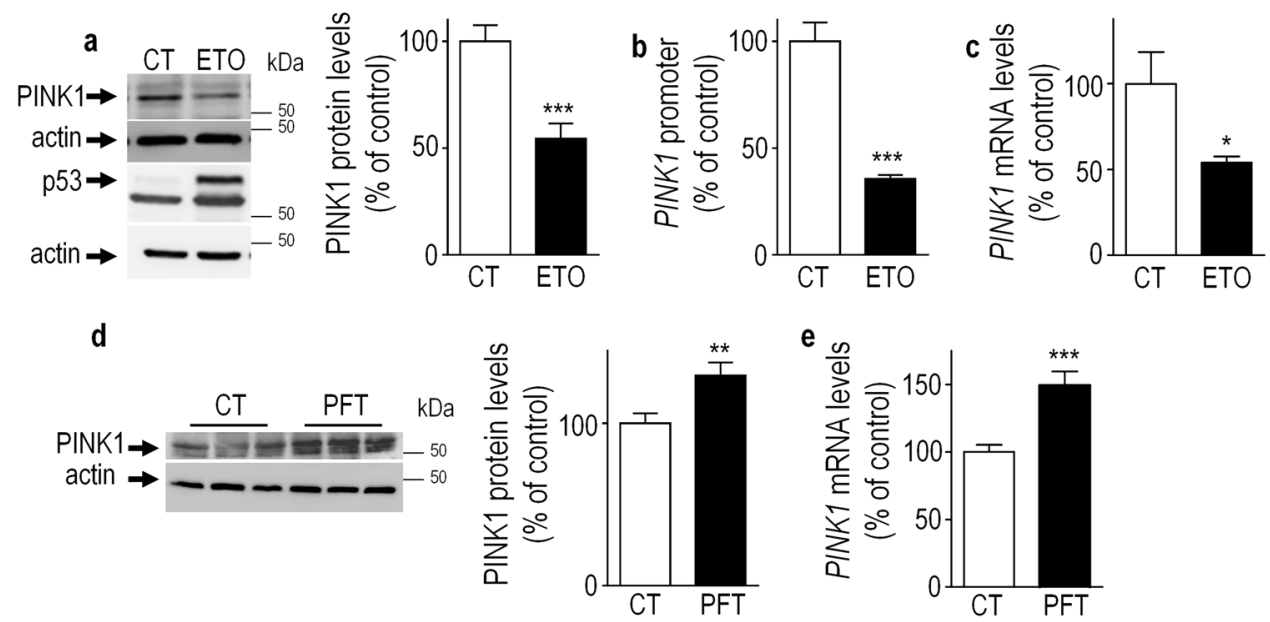

conditions, parkin and p53 control the transcription of each other via their DNA binding properties [9-11]. Parkin is also involved in the degradation of several toxic proteins by the proteasome via its ubiquitin-ligase activity [12] and was shown to be recruited to mitochondria and activated by PINK1 (PTEN-Induced Kinase 1) upon its phosphorylation $[13,14]$. In pathological conditions, the turnover of PINK1 is inhibited, leading to its stabilization in mitochondria outer membrane and parkin recruitment and phosphorylation. Consequently, activated parkin mediates the specific elimination of defective mitochondria by the lysosomal system $[15,16]$. These data linking p53 and parkin led us to hypothesize that p53 and PINK1 could be linked at a molecular level to control mitophagy.

We describe here the first pro-autophagic gene transcriptionally controlled by $\mathrm{p} 53$ in both basal and stress conditions. We demonstrate that p53 represses the transcription of the pro-mitophagic gene PINKI by several approaches. Thus, pharmacological and genetic modulation of p53 leads to a down-regulation of PINK1 transcription exvivo and in vivo. This regulation is mediated by $\mathrm{p} 53$ physical interaction with PINK1 promoter and strictly dependent of p53 nuclear subcellular localization. Finally, we show that p53-induced repression of autophagy is PINK1-dependent.

Thus, our study delineates for the first time an antiautophagic phenotype implying p53 transcriptional activity in basal and stress conditions. This novel observation may have a major impact in multiple diseases including neurodegenerative disorders and cancer where p53 function is altered and where autophagic dysfunction has been consistently documented.

\section{Results}

\section{Pharmacological modulation of p53 impacts PINK1 transcription}

We have modulated p53 expression by treating SH-SY5Y neuroblastoma human cells with etoposide (ETO), a topoisomerase II genotoxic agent that stabilizes p53 by favoring its phosphorylation [17]. As expected, we observed an ETO-induced increase in p53 expression (Fig. 1a) that was associated with a reduction of PINK1 protein expression (Fig. 1a), promoter transactivation (Fig. 1b) and mRNA levels (Fig. 1c), suggesting a p53dependent inhibitory control of PINKI transcription. We confirmed this hypothesis by assessing the effect of pifithrin- $\alpha$ (PFT), a chemical inhibitor of p53 that specifically blocks its transcriptional activity [18]. Thus, PFTinduced blockade of p53 activity increases both PINK1 protein expression (Fig. 1d) and mRNA levels (Fig. 1e). Overall, this set of data identifies p53 as a transcriptional inhibitor of PINK1 transcription. 

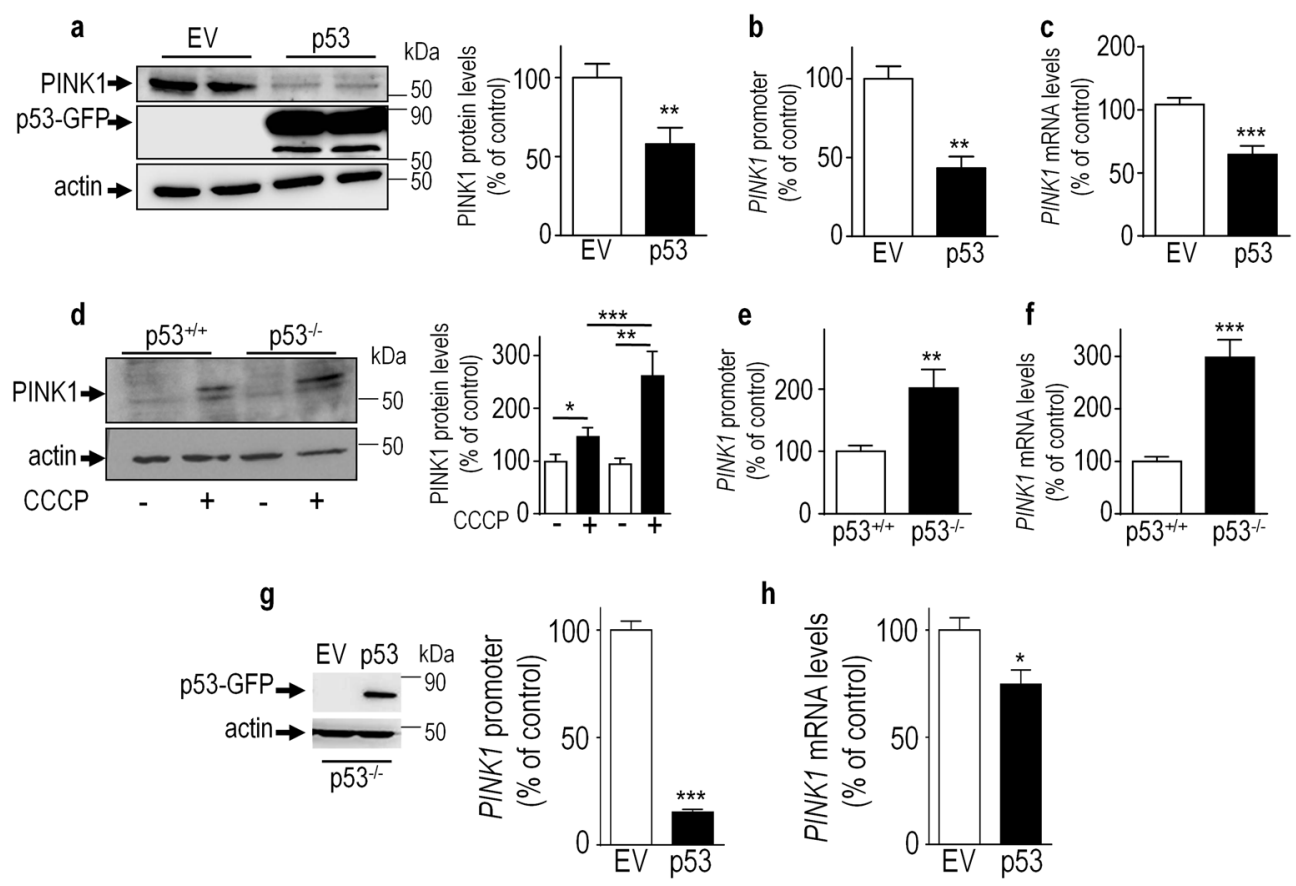

Fig. 2 Overexpressed and endogenous p53 repress PINK1 transcription. SH-SY5Y cells transiently transfected with an empty vector (EV, white bars) or wild-type p53 cDNA (p53, black bars) were assessed for PINK1 protein $(\mathbf{a}, N=8)$, promoter activation $(\mathbf{b}, N=6)$ and mRNA levels $(\mathbf{c}, N=12)$. PINK1 protein levels in basal $(-$, white bars) and CCCP $(+$, black bars, $10 \mu \mathrm{M} / 6 \mathrm{~h})$ stress conditions were analyzed in control (p53 $3^{+/+}$, black bars) or p53-deficient (p53 $3^{--}$, white bars) HCT116 cells $(\mathbf{d}, N=12)$. PINK1 promoter transactivation $(\mathbf{e}, N=6)$ and mRNA levels $(\mathbf{f}, N=12)$ were analyzed in control ( $\mathrm{p} 53^{+/+}$, black bars) or p53-deficient (p53 ${ }^{-/-}$, white bars) HCT116 cells in basal conditions as described in the Methods section. p53 and actin protein

\section{Endogenous and overexpressed p53 repress PINK1 transcription}

We aimed at examining more directly the influence of overexpressed and endogenous p53 on PINK1 transcription. Transient transfection of human wild-type p53 cDNA in SH-SY5Y cells led to drastic reductions of PINK1 protein, promoter activity and mRNA levels (compare control empty vector, (EV, white bars) and p53 (black bars) in Fig. 2a-c). The assessment of PINK1 modulation by endogenous p53 was examined in the human colorectal carcinoma cell line HCT116, where TP53 gene was genetically invalidated. We confirmed the general observations that endogenous PINK1 expression is generally low in fibroblasts and, more generally, in non-neuronal cells (See Fig. 2d, p53 ${ }^{+/+}$lane -). Thus, in this set of experiments, we took advantage of previous data, which consistently documented a stabilization of PINK1 by the stress-associated inducer of mitochondrial-depolarization-[2-(3-chlorophenyl) hydrazinylyidene] propanedinitrile (CCCP) [19]. As expected, PINK1 expression is indeed increased by CCCP in HCT116 cells (compare-and +CCCP conditions in $\mathrm{p} 53^{+/+}$lanes in levels are provided in $\mathbf{a}$ and $\mathbf{d}$ as transfection and protein charge controls. HCT116 p53-deficient $\left(\mathrm{p} 53^{-/-}\right)$cells transiently transfected with an empty vector (EV, white bars) or wild-type p53 cDNA (p53, black bars) were assessed for p53 expression (g, left panel) PINK1 promoter transactivation $(\mathbf{g}, N=8)$ and mRNA levels $(\mathbf{h}, N=6)$. Bars represent the means \pm SEM of 3 independent experiments performed in duplicates $(\mathbf{a}, \mathbf{b}, \mathbf{e}, \mathbf{g}, \mathbf{h})$ or quadruplicates $(\mathbf{c}, \mathbf{d}, \mathbf{f})$ and are expressed as percentage of control EV $(\mathbf{a}-\mathbf{c}, \mathbf{g}, \mathbf{h})$ or $\mathrm{p} 53^{+/+}$cells $(\mathbf{d}-\mathbf{f})$. Statistical analyses were performed with GraphPad Prism software by using unpaired student's $t$-test. Significant differences are: $* p<0.05$, $* * p<$ 0.01 , and $* * * * p<0.0001$.

Fig. 2d). Interestingly, p53 depletion (p53--) increases PINK1 protein expression, promoter transactivation and mRNA levels (Fig. 2d-f, black bars). In rescue experiments, we show that human $\mathrm{p} 53$ cDNA transfection in $\mathrm{p} 53^{-1-}$ HCT116 cells restore p53-associated inhibitory effect on PINK1 promoter activity (Fig. 2g) and mRNA levels (Fig. 2h). Similar phenotype was observed in an additional model of TP53 invalidation (Immortalized mouse embryonic fibroblasts invalidated for either $p 19^{a r f}$ (A-, empty bars) or for both TP53 and p19arf genes (AP-, black bars)) [20], which display increased levels of PINK1 promoter activity (Fig. S1a) and mRNA levels (Fig. S1b). Altogether, combined genetic and pharmacological data demonstrate the role of endogenous p53 as a transcriptional repressor of PINK1.

\section{p53 controls PINK1 transcription and protein expression in vivo}

Is PINK1 also down-regulated by p53, in vivo? We have analyzed the impact of both adenoviral- overexpression (Fig. 3a, b) or invalidation (Fig. 3c, d) of p53 on PINK1 protein and mRNA levels in mice brains. Striatal 

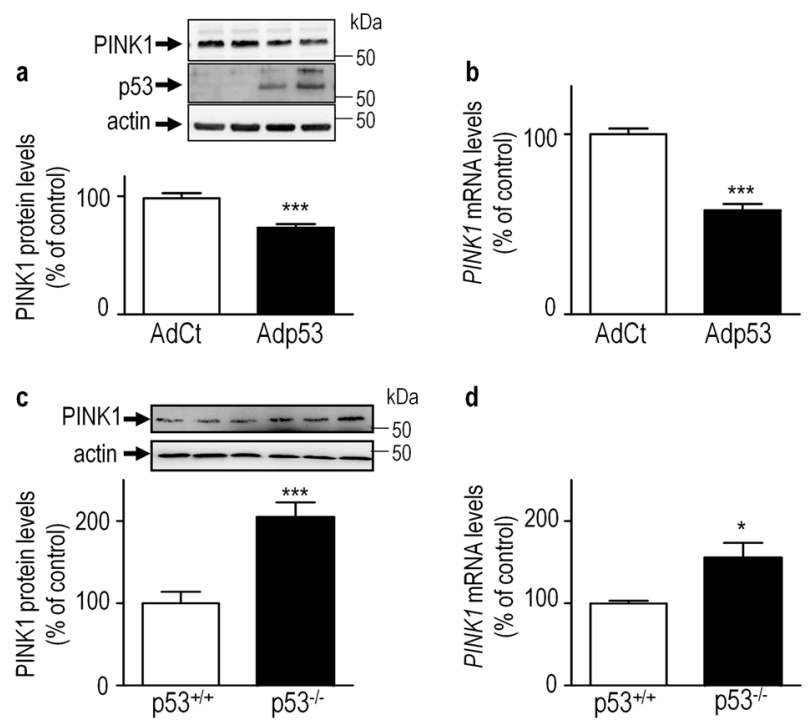

Fig. 3 p53 controls PINK1 transcription in vivo. $(\mathbf{a}, \mathbf{b})$ Effect of adenovirus-mediated wild-type p53 (Adp53, black bars) overexpression on PINK1 protein $(\mathbf{a}, N=8)$ and mRNA $(\mathbf{b}, N=8)$ levels compared to control virus (AdCt, white bars) in vivo as described in the Methods. Analyses of PINK1 protein $(\mathbf{c}, N=6)$ and mRNA levels $(\mathbf{d}, N=8)$ in control (p53 $3^{+/+}$, white bars) or TP53 gene invalidated (p53 $3^{-1-}$, black bars) in mouse brain samples as described in the Methods. Representative gels of PINK1, p53 (virus transduction efficiency) and actin (protein charge control) protein levels are provided in upper panels a and c. Bars represent the means \pm SEM of 2 independent experiments performed in quadruplicates and are expressed as percentage of control (AdCt, $\mathrm{p} 53^{+/+}$) mouse brain samples. Bars represent the means \pm SEM of 3 or 4 independent experiments performed in duplicate (c,d, respectively) and are expressed as percentage of control (AdCt, p53 ${ }^{+/+}$) mouse brain samples. Statistical analyses were performed with GraphPad Prism software by using unpaired Student's t-test. Significant differences are: $* p<0.05$, and $* * * p<$ 0.001 .

overexpression of p53 decreases PINK1 protein (Fig. 3a) and mRNA (Fig. 3b) levels. Conversely, depletion of endogenous p53 increases PINK1 protein (Fig. 3c) and mRNA (Fig. 3d) levels. It should be noted that p53 invalidation impacts neuronal PINK1 transcription and protein expression, in vivo, in absence of any stress. This data confirms the fact that endogenous level of PINK1 protein remains under thresholds of detection in non-neuronal cellular models (thus requiring CCCP treatment to unravel its expression, Fig. 2a) but not in a neuronal context. Overall, our data show that endogenous p53 negatively controls PINK1 protein and mRNA levels in vivo, thus confirming our observations gathered in various cellular models (Figs. 1 and 2).

\section{Identification and validation of a p53 binding site on PINK1 promoter}

In order to map the putative physical domain of interaction between p53 and PINK1 promoter, we have analyzed the effect of p53 expression (see Fig. 4a, middle) on full-length
(FL) and 5'end-truncated constructs of PINK1 mouse promoter driving luciferase expression (Fig. 4a, upper). p53 represses PINK1 transcription whatever the promoter construct used (Fig. 4a, lower), indicating that at least one putative functional p53 consensus binding motif was located in the shorter $0,4 \mathrm{~kb}$ construct. In silico examination of this sequence, led to the identification of at least two putative $(-296 /-285$ and $-167 /-154)$ p53 responsive elements (Fig. 4b, upper). Since the $-167 /-154$ (CCAGctgcacCAAG) sequence fits better with the canonical consensus p53 responsive element (CxxG-N6-CxxG), we have deleted the $-167 /-164$ CCAG motif from the 0.4 $\mathrm{kb}$ sequence (construct referred to as $0.4 \Delta$ ) and examined the impact of this deletion on p53-dependent PINK1 promoter activity. This deletion significantly reduced the p53-dependent decrease of PINK1 promoter activity observed with wild-type 0.4 construct (compare black bars in Fig. 4b, lower). This data validates the $-167 /$ -154 sequence as a functional p53 responsive element and confirms the ability of p53 to directly repress PINK1 transcription. However, this deletion did not fully abolish p53-associated inhibitory effect (Fig. 4b, lower). This could indicate a potential participation of the less conserved responsive element located at $-296 /-285$. To validate the physical interaction of p53 with the $-167 /-154$ PINK1 promoter region in physiological conditions, we have performed chromatin immunoprecipitation assay (ChIP). Real-time PCR assessment (Fig. 4c upper) of ChIP enrichment of PINK1 promoter DNA using specific p53 immunoprecipitant antibodies (CM1, Fig. 4c middle) and negative control IgG in both control (A-) and p53 (AP-) invalidated cells indicates a physical interaction of $\mathrm{p} 53$ with PINK1 promoter in cells harboring TP53 (A-), but not in cells devoid of TP53 (AP-, Fig. 4c, lower).

\section{Repression of PINK1 transcription requires nuclear localization of p53}

As stated in the introduction, sub-cellular localization of p53 conditions its transcription factor properties and by consequence its pro- or anti-autophagic function [2]. We have investigated the impact of the pharmacological and genetic modulation of p53 subcellular localization on PINK1 transcription. Leptomycin B (LM), specifically and potently inhibits the CRM1/exportin 1 pathway of nuclear export by directly binding the CRM1 protein $[21,22]$ and was shown to block p53 in the nucleus [23]. The impact of LM on p53 translocation was confirmed in Fig. 5a by immunofluorescence imaging. Thus, as expected, LM treatment blocks p53 in the nucleus as evidenced by the exclusive colocalization between p53 and nucleus marker DAPI (compare merge DAPI/p53 in control (CT) versus LM conditions). Importantly, LM treatment triggers a 
a

Mouse PINK1 promoter
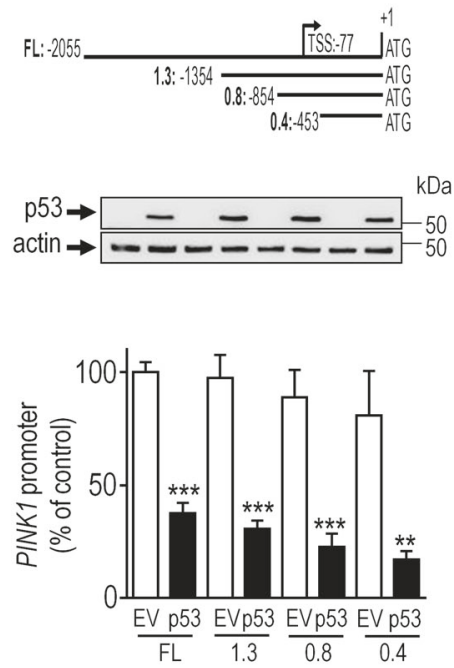

Mouse PINK1 promoter

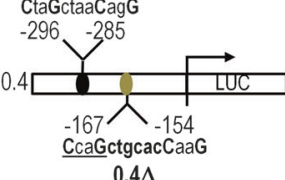

$0.4 \Lambda$
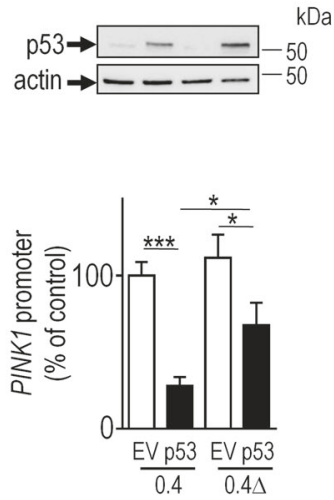

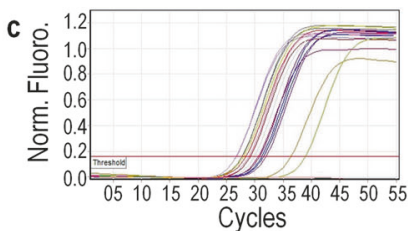
Cycles

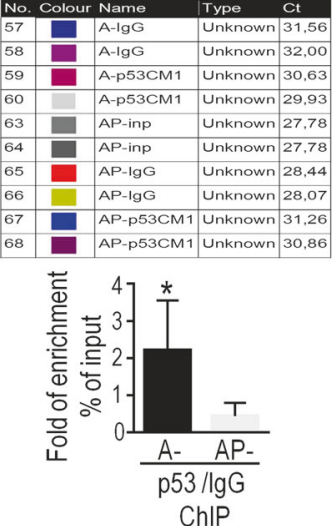

Fig. 4 Mapping and validation of p53 binding domain of PINK1 promoter. Upper panel a $(N=12)$ represents the 5 'end deletion constructs of the full-length (FL) mouse PINK1 promoter region. The PINK1 promoter constructs described in a (upper scheme) were co-transfected with the $\beta$-galactosidase reporter gene and either empty vector (EV, white bars) or wild-type p53 (p53, black bars) cDNA in HEK293 cells and then luciferase activity was measured as described in Methods. Upper panel b represents the two potential p53-responsive elements (-296/-285 and -167/-154) on the shorter 0.4Kb-luciferase PINK1 promoter construct. In lower panel $(\mathbf{b}, N=12)$, PINK1 promoter constructs $0.4 \mathrm{~Kb}$ wild-type $(0.4)$ or deleted from the $-167 /$ 164 sequence $(0.4 \Delta)$ were co-transfected with the $\beta$-galactosidase reporter gene and either empty cDNA (EV, white bars) or wild-type p53 (p53, black bars) cDNA, then luciferase activity was measured as described in Methods. Representative gels of p53 expression and actin

drastic reduction of PINK1 protein expression (Fig. 5b), promoter activity (Fig. 5c) and mRNA levels (Fig. 5d), indicating that the control of PINK1 transcription is linked to p53 nuclear localization. Several works have shown that nuclear p53 exerts a pro-autophagic function while cytoplasmic p53 triggers an anti-autophagic phenotype. Thus, we were facing a case where PINK1 could well behave as the first pro-autophagic gene transcriptionally repressed by nuclear p53. To strengthen this hypothesis, we designed constructs encoding either wild-type p53 coupled to GFP (WT) or harboring two mutations in its previously delineated nuclear export (GFP-p53 L22Q, W23S, NES ${ }^{\mathrm{m}}$ ) or nuclear import (GFP-p53 KKK280-282AAA, NLS ${ }^{\mathrm{m}}$ ) domains $[23,24]$ and we examined their impacts on PINK1 transcriptional regulation in SH-SY5Y cells. Immunohistochemical analyses of GFP-tagged p53 constructs confirmed that the NES and NLS mutations indeed blocked p53 in the nucleus (Fig. 5e, middle) and cytoplasm (Fig. 5e, lower), respectively. Of most interest, nuclear p53 decreased PINK1 expression (Fig. 5f, upper) PINK1 promoter activation and mRNA levels $\left(\mathrm{NES}^{\mathrm{m}}\right.$, black bars in as transfection efficiency and gel charge controls are provided in $\mathbf{a}$ and b. Bars represent the means \pm SEM of 4 independent experiments performed in triplicates and are expressed as percentage of control (FL, 0.4) EV transfected cells. Upper panel c represents real-time PCR curves of samples from a standard ChIP experience (c, middle panel) performed in control (A-, black bars) and TP53-invalidated MEF cells (AP-, gray bars). Lower panel $\mathbf{c}$ represents the quantification analysis of the ChIP enrichment of PINK1 promoter using and anti-p53 antibody (anti-CM1) over that of control IgG. Bars represent the means \pm SEM of 2 independent experiments performed in triplicate and are expressed as fold of enrichment according to the formula described in the methods. Statistical analyses were performed with GraphPad Prism software by using unpaired Student's $t$-test. Significant differences are: $* p<0.05, * * p<0.01, * * * p<0.001$.

Fig. 5f, g) while sequestration of p53 in the cytoplasmic compartment fully abolished p53-induced reductions of PINK1 protein expression (Fig. 5f upper), promoter activity and mRNA levels $\left(\mathrm{NLS}^{\mathrm{m}}\right.$, gray bars in $5 \mathrm{f}, \mathrm{g}$ ). This data firmly demonstrates that PINK1 transcriptional repression requires exclusive nuclear localization of p53 and thus, unravels PINK1 as the first pro-autophagic gene transcriptionally repressed by $\mathrm{p} 53$. This is the very first demonstration of an anti-autophagic phenotype linked to nuclear p53 transcription factor function.

\section{p53 represses mitophagy ex-vivo and in vivo}

The pro-mitophagy response of PINK1 is associated to increased LC3 maturation, decreased expression of mitophagy markers like TIM23, TOM20 and HSP60 and modulation of p62, optineurin and NDP52 autophagy receptors [25-27]. From our data indicating a p53-mediated repression of PINK1, one should expect that TP53 invalidation mimics PINK1-related pro-autophagy response. As shown in Fig. 6, full TP53 invalidation (see abolishment of p53 

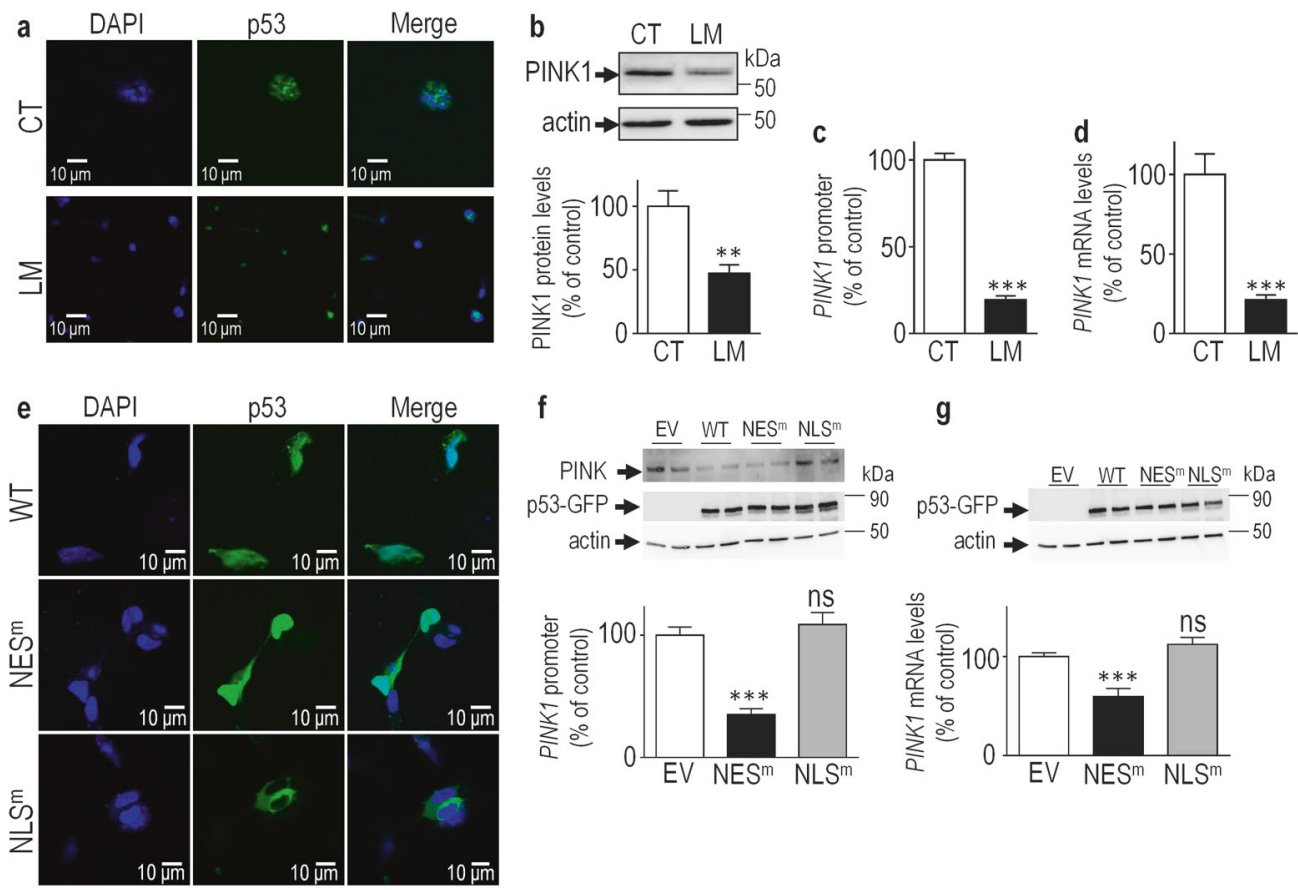

g
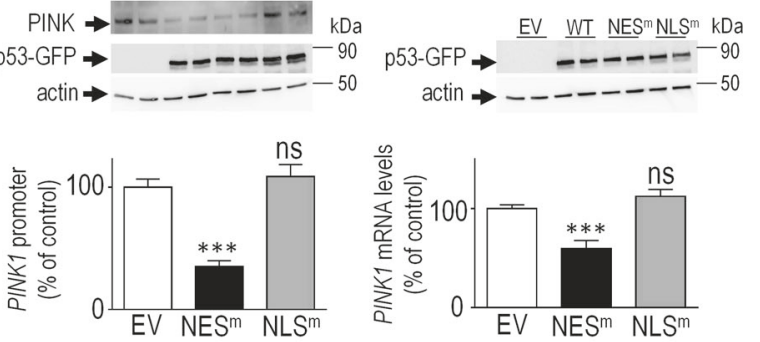

Fig. 5 Impact of the pharmacological and genetic modulation of p53 localization on PINK1 transcription. (a) Immunohischemical detection of endogenous p53 and DAPI nuclear labeling in SH-SY5Y cells treated with vehicle (CT) or leptomycin (LM) as described in the methods. (b-d) PINK1 protein expression $(\mathbf{b}, \mathbf{f}, N=12)$, promoter activation $(\mathbf{c}, N=6)$ and mRNA levels $(\mathbf{d}, N=9)$ were analyzed in SH-SY5Y cells treated with vehicle (CT) or with leptomycin (LM) as described in the Methods. (e) Immunohistochemical analysis of GFPtagged p53 constructs versus DAPI nuclear labeling showing the presence of wild-type p53 (WT) in the nucleus and cytoplasm, p53 NES mutant (L22Q, W23S p53, NES ${ }^{\mathrm{m}}$ ) in the nucleus and p53 NLS mutant $\left(\mathrm{K} 280-282 \mathrm{~A} \mathrm{p} 53, \mathrm{NLS}^{\mathrm{m}}\right)$ in the cytoplasm. (f,g) SH-SY5Y

expression in 6a) increases PINK1 expression levels (Fig. 6a, b), LC3 maturation (Fig. 6a, c) optineurin (Fig. 6a, h) and NDP52 expressions (Fig. 6a, i) while it decreases the levels of p62 (Fig. 6a, d) and the mitophagy markers TIM23 (Fig. 6a, e), TOM20 (Fig. 6a, f) and HSP60 (Fig. 6a, g). These observations fully mimic the PINK1-associated promitophagy phenotype previously described. Importantly, TP53 knockout mice brain analysis corroborates this conclusion. Thus, Fig. 7 shows that TP53 invalidation increased levels of LC3-2 (Fig. 7a) and optineurin (Fig. 7d) and reduced p62 (Fig. 7b) and TIM23 (Fig. 7c) expressions. In addition, we have also carried out ShRNA inactivation of TP53 by transient transfection in SH-SY5Y cells. Our data indicate that in the neuronal dopaminergic cell system, again p53 modulates p62, HSP60; TIM23 and TOM20 similarly to HAP cells and mice brain (data not shown).

The control of mitophagy is tightly linked to PINK1 and parkin interplay. Thus, parkin itself represses TP53 [9], indirectly increases PINK1 transactivation [25] and is controlled by $\mathrm{p} 53[10,11]$. This intricate functional network led cells transiently transfected with empty vector (EV), wild-type p53 (WT), p53 NES mutant $\left(\mathrm{NES}^{\mathrm{m}}\right)$, or p53 NLS mutant $\left(\mathrm{NLS}^{\mathrm{m}}\right)$ and then assessed for PINK1 promoter activity $(\mathbf{f}, N=12)$ and mRNA levels $(\mathbf{g}$, $N=12)$. p53 (f,g) and actin $(\mathbf{b}, \mathbf{f}, \mathbf{g})$ immunoreactivities are provided as read-out of p53 activation and gel loading respectively. Bars represent the means \pm SEM of 3 independent experiments performed in duplicates $(\mathbf{c})$ or quadruplicates $(\mathbf{b}, \mathbf{f}, \mathbf{g})$ and are expressed as percentage of vehicle-treated cells (b-d) or EV control cells (e-g). Statistical analyses were performed with GraphPad Prism software by using unpaired Student's $t$-test. Significant differences are: $* * p<0.01$, $* * * * p<$ 0.0001 and ns, non-significant.

us to question the putative implication of parkin in the regulation of PINK1 by p53. We have treated control and parkin knockout cells with PFT and evaluated the influence of this p53 transcriptional inhibitor on PINK1 mRNA levels. In agreement with our previous studies [9, 25], we confirmed that parkin deficiency lowers PINK1 mRNA levels (compare black bars in Supplementary Fig. S2a) and increases p53 mRNA levels (compare black bars in Supplementary Fig. S2b). However, PFT did not prevent parkin-mediated PINK1 regulation (compare grey bars in Supplementary Fig. S2a) thus indicating that PINK1 regulation by $\mathrm{p} 53$ is parkin independent.

\section{Negative control of mitophagy by p53 is dependent of PINK1}

The similar phenotypes triggered by TP53 invalidation (Figs. 6 and 7) and PINK1 expression [25-27] strongly support our view of a p53-dependent control of mitophagy via PINK1 repression. In order to definitely confirm this hypothesis, we have assessed the contribution of PINK1 to 


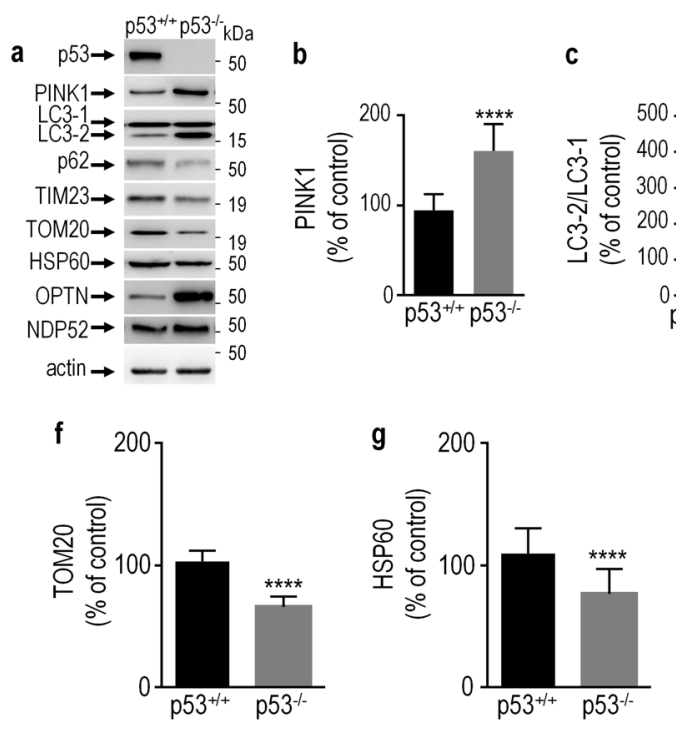

Fig. 6 TP53 knockout triggers a pro-mitophagic response. PINK1 (a, b, $N=8)$, LC3-2/LC3-1 (a,c, $N=8)$, p62 (a,d, $N=12)$, TIM23 (a,e, $N=10)$, TOM20 (a,f, $N=9)$, HSP60 (a,g, $N=16)$, optineurin $(\mathbf{a}, \mathbf{h}$, $N=9)$ and NDP52 (a,i, $N=8)$ protein levels were analyzed in control $\left(\mathrm{p} 53^{+/+}\right)$or TP53-deficient $\left(\mathrm{p} 53^{-/-}\right.$) HAP1 cells as described in the Methods. Bars represent the means \pm SEM of 3-4 independent

p53-linked mitophagic phenotype. Thus, we treated human haploid (HAP) cells harboring $\left(\mathrm{PINK}^{+/+}\right.$) or lacking (PINK ${ }^{-/}$) endogenous PINK1 with PFT. First, in agreement with previous data $[7,8]$ the blockade of endogenous $\mathrm{p} 53$ by PFT increases autophagic markers similarly to CCCP (CP), the mitochondrial uncoupler usually used to unravel PINK1 mitophagy response. Thus, PFT and CP-treated PINK1 ${ }^{+/+}$ cells display increased expressions of LC3-2 (Figs. 8a, b) and decreased levels of p62 cargo protein (Figs. 8a, c) and mitochondrial markers TIM23 (Figs.8a, d), TOM20 (Figs. 8a, e) and HSP60 (Figs. 8a, f) confirming that pharmacological impairment of p53 activity leads to a promitophagy phenotype. As expected PINK $1^{-1-}$ cells show a decrease of LC3 maturation (Figs. 8a, b), concomitant to an increase of p62 (Figs. 8a, c), TIM23 (Figs. 8a, d), TOM20 (Figs. 8a, e) and HSP60 (Figs. 8a, f) protein levels when compared to PINK1 ${ }^{+/+}$cells in stress (CP and PFT) conditions. Of most interest, PFT-mediated effect on these autophagic/mitophagic markers was fully abolished by PINK1 depletion (Fig. 8a-f). This data firmly comfort our hypothesis of PINK1-dependent p53-associated repression of mitophagy.

\section{Discussion}

Several works underlined a duality in the ability of p53 to control autophagic response $[7,8,28]$. Thus, both p53 activation and depletion trigger increased autophagy. It is d

e

d
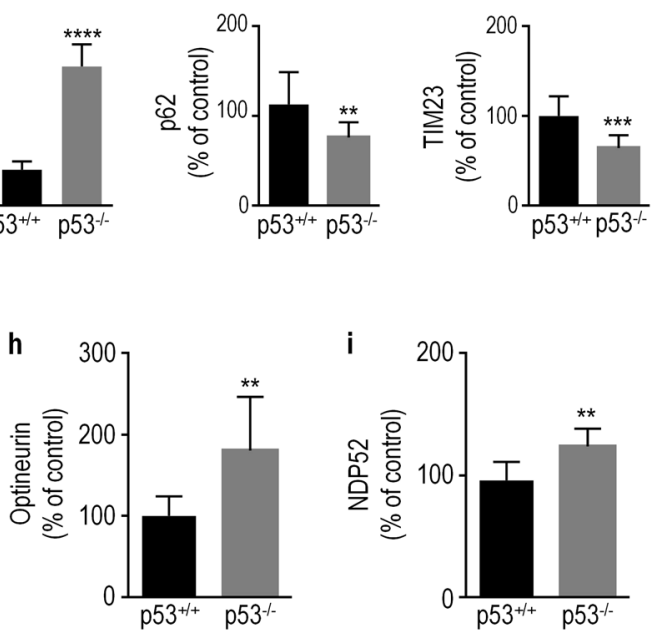

experiments performed in triplicate and are expressed as percent of

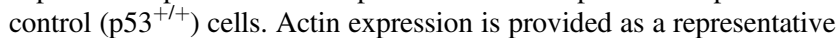
gel loading control in a. Statistical analyses were performed with GraphPad Prism software by using unpaired Student's $t$-test. Significant differences are: $* * \quad p<0.01, \quad * * * p<0.001$ and $* * * * p<0.0001$.

consensually admitted that genotoxic stress or oncogenic activation triggers a p53-dependent pro-autophagic phenotype that is mostly due to nuclear p53 transcriptional activity [3, 4]. Conversely, p53-linked anti-autophagic response was reported to be independent of its ability to regulate gene transcription but rather linked to cytoplasmic p53. Thus, it was shown that several autophagic inducers enhanced the proteasomal degradation of $\mathrm{p} 53$ and thereby, down-regulate the p53-mediated anti-autophagic phenotype [7]. Furthermore, the work of Tasdemir et al. has demonstrated that endogenous p53 could repress autophagy via the inhibition of AMPK and activation of mTOR [7]. This conclusion was supported by the observation that p53, in which nuclear export domain was mutated, exclusively resides in the nucleus and was unable to repress autophagy. Overall, this work proposed that the p53 anti-autophagic effect is exclusively linked to its cytoplasmic localization [7]. Our work however indicates that the situation is likely not so simplistic. Thus, we clearly demonstrated by combined and complementary approaches that p53 can also repress autophagy, ex-vivo and in vivo, via the transcriptional repression of PINK1, a well characterized proautophagic effector [29]. Thus, nuclear p53 can also trigger an anti-autophagic phenotype. The discrepancy between our work and that of Tasdemir's may be linked to the fact that these authors investigated the impact of nuclear $\mathrm{p} 53$ on "non-selective" macro-autophagy and several players of the AMPK/mTOR axis. Our data show that nuclear p53 may thus have an additional role on PINK1-dependent control of 
a
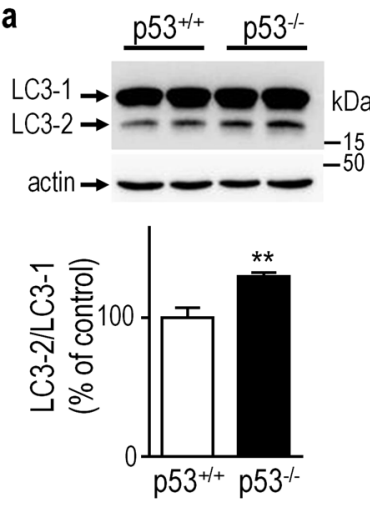

C
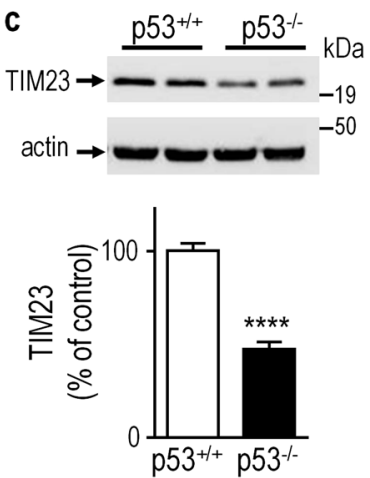

b
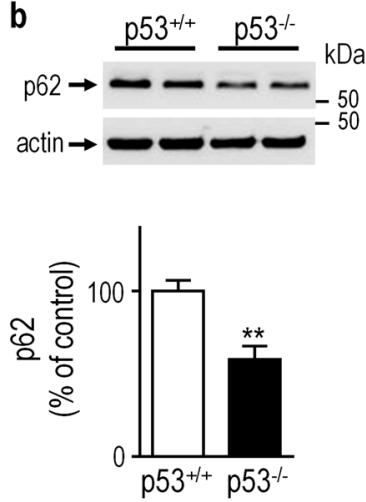

d
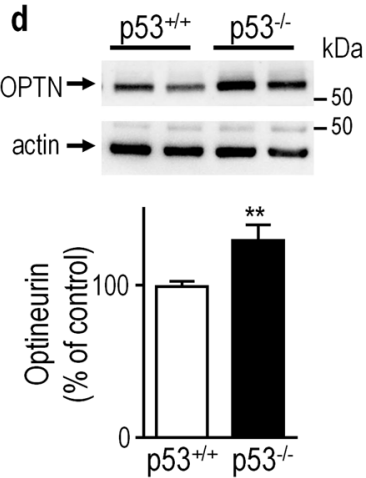

Fig. 7 Evaluation of mitophagy control by p53 in mice brain. LC3-2/ LC3-1 (a, $N=9)$, p62 (b $N=9)$, TIM23 (c, $N=9)$ and optineurin $(\mathrm{OPTN}, \mathbf{d}, N=9)$ protein levels were analyzed in control $\left(\mathrm{p}^{2} 3^{+/+}\right)$or TP53-deficient $\left({\mathrm{p} 53^{-l-}}^{-}\right)$knockout mice brains as described in the Methods. Bars represent the means \pm SEM of 3 independent experiments performed in triplicate and are expressed as percent of control $\left(\mathrm{p} 53^{+/+}\right)$cells. Actin expression is provided as a representative gel loading control in a. Statistical analyses were performed with GraphPad Prism software by using unpaired Student's $t$-test. Significant differences are: $* * p<0.01, * * * p<0.001$.

"selective" mitochondrial autophagy. Furthermore, since transcriptional regulation may be cell type specific, one cannot exclude the possibility that in Tasdemir et al., the use of p53-deleted human colon adenocarcinoma cells could have precluded the delineation of additional p53-dependent transcriptional pathways repressing autophagy.

Several lines of evidence indicate that mitophagy could well be controlled by an intricate functional interplay linking parkin, p53 and PINK1. First, there exists a feed-back loop by which, parkin transrepresses p53 [9], which conversely, up-regulates parkin transcription [11]. This interplay has functional consequences where PINK1 plays a key role. Thus, cytoplasmic p53 affects parkin translocation to the mitochondria, enabling its interplay with PINK1 and thereby, elimination of malfunctioning mitochondria by mitophagy [30]. Numerous studies have documented the functional relationship between parkin and PINK1. This led to the consensus that parkin acts as a privileged downstream effector of a cascade by which PINK1 controls

mitochondrial homeostasis and elimination of flawed mitochondria by selective autophagy [31]. Breaking this dogma, we have recently shown that parkin could also act upstream to PINK1 to regulate its transcription and promitophagic function [25]. Thus, one could envision a cascade in which p53 repression by parkin would ultimately lead to PINK1 up-regulation. However, we demonstrate here that PINK1 regulation by p53 is independent of parkin, thus suggesting the existence of an alternative interplay between these proteins controlling mitophagy. This scenario where PINK1 could modulate mitophagy independently of parkin has been demonstrated in previous works (for review see 27). Overall, this data highlight multiple pathways by which p53 could control mitophagy, including yet unraveled additional function of nuclear p53 as an autophagic repressor via transcriptional repression of PINK1.

Since p53 expression and function are altered in both neurodegenerative diseases and cancer and given the fact that both pathologies are characterized by drastic alterations of autophagic processes, one can envision that either gain (neurodegeneration) or loss (tumorigenicity) of function of p53 could directly influence PINK1 function and thereby, could contribute to the anatomical stigmata and clinical pictures observed in these pathologies.

\section{Materials and methods}

\section{Cellular and animal models}

Mouse embryonic fibroblasts (MEF), Human Colorectal adenocarcinoma (HCT116), SH-SY5Y human neuroblastoma and human embryonic kidney (HEK293) cell lines were cultured in Dulbecco's modified Eagle's medium supplemented with $10 \%$ fetal calf serum, penicillin (100 U/ $\mathrm{ml})$ and streptomycin $(50 \mu \mathrm{g} / \mathrm{ml})$ purchased from Life Technologies (CA, USA) and incubated at $37^{\circ} \mathrm{C}$ in a $5 \%$ $\mathrm{CO}_{2}$ atmosphere. Immortalized mouse embryonic fibroblasts invalidated for TP53 or for TP53 and p1 $9^{\text {arf }}$ genes were kindly provided by Dr. M. Roussel (St. Jude Children's Research Hospital Memphis, TN, USA) whereas the human colorectal carcinoma cell lines HCT116 invalidated or not for TP53 were provided by Dr. JC Bourdon (University of Dundee, Dundee, UK). MEF cells invalidated for the PARK2 (parkin) gene were kindly provided by Dr. T. Dawson (Johns Hopkins University School of Medicine, Baltimore, MD, USA.). HAP1 cell line, purchased from Horizon Genomics (UK), is a haploid human cell line that was derived from KBM-7 cells [32]. HAP1 clones HZGHC000798c008 (HAP $\mathrm{PINK}^{-1-}$ ) and HZGHC001068c001 (HAP p53 ${ }^{-l-}$ ) were engineered using CRISPR/cas9 approach. HAP1 cells were cultured in Iscove's Modified Dulbecco's Medium (IMDM) with 10\% 

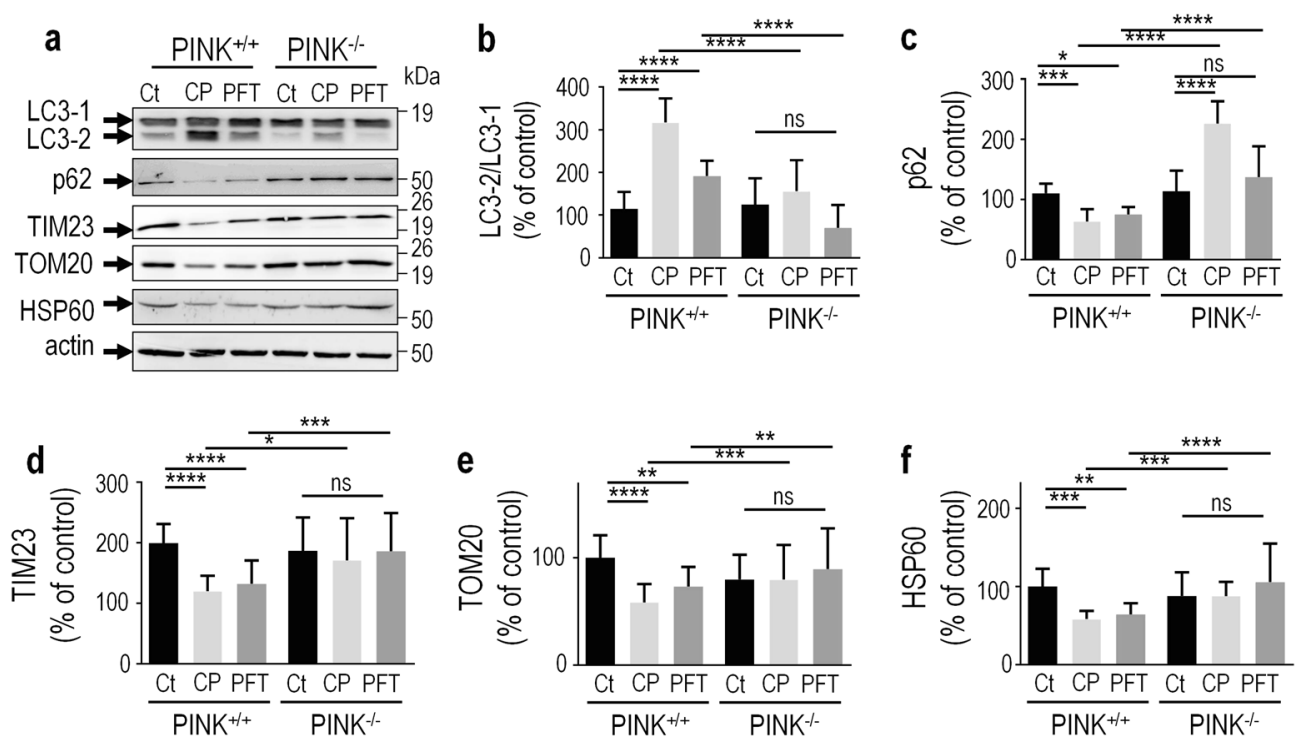

Fig. 8 p53 repression of autophagy is PINK1-dependent. LC3-2/LC3$1(\mathbf{a}, \mathbf{b}, N=12)$, p62 (a,c, $N=12)$, TIM23 (a,d, $N=12)$, TOM20 (a,e, $N=12)$ and HSP60 (a,f, $N=12)$ protein levels were analyzed in control $\left(\mathrm{PINK}^{+/+}\right)$or Pinkl-deficient $\left(\mathrm{PINK}^{-l-}\right)$ HAP cells treated with vehicle $(\mathrm{Ct})$, pifithrin- $\alpha$ (PFT, $30 \mu \mathrm{M}, 4 \mathrm{~h})$ or CCCP $(\mathrm{CP}, 10 \mu \mathrm{M}$, $6 \mathrm{~h})$ as described in the Methods. Bars represent the means \pm SEM of 4

fetal calf serum, penicillin $(100 \mathrm{U} / \mathrm{ml})$ and streptomycin $(50$ $\mu \mathrm{g} / \mathrm{ml}$ ) purchased from Life Technologies (CA, USA). TP53 knockout mice have been provided by Dr. M. Serrano (Spanish National Cancer Research Center, Madrid, Spain). Human cell lines from ATTC were validated by STR profiling method according to manufacturer's instruction (geneprint ${ }^{\circledR} 10$ system, Promega, France) and routinely tested for mycoplasma contamination.

\section{Pharmacological modulation of p53}

The pharmacological modulation of $\mathrm{p} 53$ was obtained after incubations with etoposide $(16 \mathrm{~h}, 150 \mu \mathrm{M})$, pifithrin- $\alpha$ $(4-16 \mathrm{~h}, 10-30 \mu \mathrm{M})$ leptomycin $(16 \mathrm{~h}, 10 \mathrm{nM})$ purchased from Sigma (MO, USA). The pharmacological modulation of PINK1 was obtained after $6 \mathrm{~h}$ incubations with CCCP $(10 \mu \mathrm{M})$ purchased from Sigma. After treatments, cells were recovered and protein and RNA analyses were monitored as described below. For the analysis of the impact of these drugs to PINKI promoter activity, cells were transiently transfected with the promoter of PINK1 driving the luciferase reporter gene expression (see Luciferase-based reporter assays section) and $24 \mathrm{~h}$ post-transfection submitted to the above described treatment.

\section{Immunohistochemical analysis}

SH-SY5Y cells were cultured on glass coverslips in $35 \mathrm{~mm}$ dishes, transiently transfected with empty vector, wild type or independent experiments performed in triplicates and are expressed as percent of control HAP1 $\left(\mathrm{HAP}^{+/+}, \mathrm{Ct}\right)$ cells. Actin expression is provided as a representative gel loading control in a. Statistical analyses were performed with GraphPad Prism software by using unpaired Student's $t$-test. Significant differences are: $n s$ not significant, $* p<0.05, * * p<0.01$ and $* * * p<0.001$.

mutated p53 cDNA by means of lipofectamine and then fixed with $1.5 \%$-formaldehyde for $20 \mathrm{~min}$, washed three times in PBS and incubated with anti-p53 CM1 antibodies for two hours. After three additional washes with PBS, cells were incubated with goat anti-rabbit secondary antibody conjugated to Alexa Fluor-488 (Molecular Probes, MA, USA) for $1 \mathrm{~h} .300 \mathrm{nM}$ DAPI (Roche Diagnostics S.A.S, France) was added (with PBS in order to stain the nuclei. Coverslips were then mounted in Vectashield mounting medium (Vector Laboratories LTD, UK) and staining was visualized with a confocal microscope. The same protocol was used when cells were cultured on glass coverslips in $35 \mathrm{~mm}$ dishes and treated with vehicle (DMSO) or leptomycin $(10 \mathrm{nM}, 16 \mathrm{~h})$.

\section{Western-blot protein analysis}

Cells and mouse brains were lysed in Prusiner's buffer (Tris-HCl 1M; pH 7.5 containing $\mathrm{NaCl}(150 \mathrm{mM})$, EDTA $(5 \mathrm{mM})$, Triton X100 $(0.5 \%)$, deoxycholate and protease inhibitor cocktail) and the homogenates obtained briefly sonicated. Aliquots of $50 \mu \mathrm{g}$ of total protein were loaded to (8-16\%) SDS-PAGE gels. After migration, proteins were wet-transferred to Hybond $\mathrm{C}$ membranes (GE Healthcare Europe $\mathrm{GmbH}$, France) and immunoblotted using the following antibodies: anti-PINK1 C-terminal (BC100-494, Novus Biologicals, France) and (AC-R3173-2, Abiocode, CA,USA); anti-p53 (CM1, kindly provided by J.C. Bourdon); anti-LC3 (NB100-2220, Novus Biologicals); antip62/SQSTM1 (NBP1-49956, Novus Biologicals); anti- 
TIM23 (611222, BD Biosciences, USA); anti-TOM20 (612278, BD Biosciences, USA), anti-HSP60 (sc-59567, Santa Cruz Biotechnology Inc., Germany), anti-optineurin (sc-166576, Santa Cruz Biotechnology Inc., Germany), anti-NDP52 (sc-376540, Santa Cruz Biotechnology Inc., Germany) and anti-actin (clone AC-74, A5316, Sigma) antibodies. Immunological complexes were revealed with either anti-rabbit or anti-mouse IgG-coupled peroxidase antibodies (Jackson ImmunoResearch, UK) by the electrochemiluminescence detection method (Roche Diagnostics S.A. $\mathrm{S}$, France). Chemiluminescence was recorded using a luminescence image analyzer LAS-4000 (Raytest, Fuji, France) and quantifications of non-saturated images were performed with the FUJI Film Multi Gauge image analyzer software.

\section{Plasmid constructs and ex-vivo transfection}

Human and mouse full length and mouse 5 'end-truncated PINK1 promoter-luciferase constructs have been previously described [33]. The pGL3 vector containing the mouse PINK1 promoter served as a template to generate the promoter deleted of the 5'-CCAG-3' nucleotides. This deleted motif constitutes part of the $5^{\prime}$ end half-site of p53 putative binding site, 5'-CCAGctgcacCAAG-3', located from nucleotides -167 to -154 upstream of mPINK1 ATG start codon. The primers used were 5'- GGT TCA AAG TGC AAA CTG CAC CAA GGG ATG - $3^{\prime}$ (forward primer) and 5'- CAT CCC TTG GTG CAG TTT GCA CTT TGA ACC $-3^{\prime}$ (reverse primer). The plasmids GFP-p53 and GFP-p53 NES were a gift from Tyler Jacks (Addgene plasmids \# 12091 and 12092). We used the GFP-p53 vector to generate with the mutagenesis kit QuickChange II (Stratagene, CA, USA) the GFP-p53NLS mutant (mutation of the 280-282 KKK sequence of p53 nuclear localization signal (NLS) in AAA). The following forward and reverse mutagenesis primers: 5'-TCC TCT CCC CAG CCA GCG GCG GCA CCA CTG GAT GGA GAA TAT- ${ }^{\prime}$ ' and 5'-ATA TTC TCC ATC CAG TGG TGC CGC CGC TGG CTG GGG AGA GGA-3' purchased from Eurogentec (France) were used. All the constructs were verified by full sequencing. Transient transfections of the various cells systems cells were carried out by means of lipofectamine 2000 (Life Technologies) according to the manufacturer's instructions.

\section{Luciferase-based reporter assays}

The transactivation of the wild-type (human and mouse) and mutated PINK-1 mouse promoter described in the plasmid constructs section was followed by recording the luciferase reporter gene activity $24 \mathrm{~h}$ after co-transfection of $1 \mu \mathrm{g}$ of the above cDNAs and $0,5 \mu \mathrm{g}$ of $\beta$-galactosidase cDNA (in order to normalize for transfection efficiencies) by means of Luciferase and $\beta$-galactosidase enzyme assays systems according to the manufacturer's (Promega) instructions. When necessary, in a subset of experiments, $0,5-1 \mu \mathrm{g}$ of empty pcDNA3.1, wild type or mutated p53 were co-transfected.

\section{RNA extraction, reverse transcription and real-time PCR analysis}

RNA from cells and RNA later (Qiagen, Germany) stabilized mouse brains were extracted and treated with DNAse using RNeasy or RNeasy Plus Universal Mini kits respectively following manufacturer's instructions (Qiagen). Two $\mu \mathrm{g}$ of total RNA were reverse transcribed (GoScript Reverse Transcriptase, Promega) using Oligo-dT priming. Then, samples were subjected to real-time PCR by means of a Rotor-Gene 6000 apparatus (Qiagen), using the SYBR Green detection protocol. Gene-specific primers were designed with the Universal Probe Library Assay Design Center software (Roche Applied Science, France). Relative expression levels of human PINK-1 (forward 5'-CGA GGA ACT CGT TTG AAG GG-3'; reverse 5'-CCA GGT GGC AAA TCA GAC ATG-3'), mouse PINK-1 (forward 5'-CGC CTA TGA AAT CTT TGG GC-3'; reverse 5'-GCA CTG CCT TGG CCA TAG AA-3'), amplification products were normalized for RNA concentrations with human Topoisomerase 1 (forward 5'-CCC TGT ACT TCA TCG ACA AGG-3'; reverse 5'-CCA CAG TGT CCG CTG TTT C-3'), mouse Topoisomerase 1 (forward 5'-TGC CTC CAT CAC ACT ACA GC-3'; reverse 5'-CGC TGG TAC ATT CTC ATC AGG-3') and human GAPDH (forward 5'-AGC CAC ATC GCT CAG ACA C-3'; reverse 5'-GCC CAA TAC GAC CAA ATC C- $3^{\prime}$ ) and mouse GAPDH (forward 5'TGT CCG TCG TGG ATC TGA C-3'; reverse 5'-CGT GCT TCA CCA CCT TCT TG-3') housekeeping genes.

\section{Chromatin immunoprecipitation (ChIP) assay}

We performed ChIP according to EZ- ChIP kit instructions (Millipore). Briefly, $10^{7}$ cells were fixed with formaldehyde (1\% final concentration), treated with glycine to quench unreacted formaldehyde and recovered in cold phosphate buffered saline (PBS) containing the protease inhibitor cocktail II. Pelleted cells were resuspended in the SDS lysing buffer and sonicated on ice in order to yield chromatin fragments of about $200-500 \mathrm{bp}$ in size. After a preclearing step using protein $\mathrm{G}$ Agarose, immunoprecipitation was performed with either anti-p53 primary antibody (CM1, JC Bourdon, University of Dundee, Dundee, UK) or normal mouse $\mathrm{IgG}$ as a negative control. Immuno-complexes were then incubated with a solution of protein G-agarose. After elution of the immuno-complexes from beads and crosslinks reversal RNAse and proteinase $\mathrm{K}$ treatments aimed at eliminating remaining RNAs and proteins were performed. 
DNA was purified and subjected to a real-time PCR using primers (forward: 5'-GTT-GTT-CAC-AAC-CCC-TCGACC-TGG-G-3'; reverse: 5'-GAC-AAC-AAC-AAA-CTTCGG-GGG-CGG-C-3') specific for the -205/-47 DNA region (159 bases-long amplicon) upstream the start codon of mouse PINKI gene. We calculated the fold enrichment (FE) as the ratio of the amplification efficiency of the ChIP sample over that of the IgG ( $\mathrm{FE}=\% \mathrm{ChIP} / \% \mathrm{IgG})$. Briefly, to do so we first produced a standard curve by performing qPCR with PINK1 primers on known (10-fold serial dilutions) DNA quantities of input DNA. We then did qPCR runs of ChIP and IgG samples along with the dilution series of the input DNA standards. We created a linear regression plot with the $\mathrm{Ct}$ (Threshold Cycle) obtained and plot $\mathrm{Ct}$ versus DNA quantity (log scale) of the dilutions to generate the slope of the standard curve. We calculated the efficiency of the primers [formula: $\mathrm{E}$ (\%efficiency) $=10(-1 /$ slope $)-1$ ] and the amplification efficiency (AE) [formula: $\mathrm{AE}=10$ (-1/slope)]. We calculated the \% ChIP [formula: \% ChIP $=\mathrm{AE}$ (Input Ct-ChIP Ct)*(dilution factor) (100)] and the \% IgG [formula: \% $\mathrm{ChIP}=\mathrm{AE}($ Input $\mathrm{Ct}-\mathrm{IgG} \mathrm{Ct}) *($ dilution factor) (100)]. This classical procedure allows calculating fold enrichment (FE) [formula: $\mathrm{FE}=\% \mathrm{ChIP} / \% \mathrm{IgG}$ ].

\section{Adenovirus-mediated p53 overexpression in mouse brain}

C57BL6 mice were purchased from Charles River Laboratories (France) and maintained at $21^{\circ} \mathrm{C}$ on a $12 \mathrm{~h}$ light and $12 \mathrm{~h}$ dark cycle. Animal care procedures were in accordance with the guidelines established by the European Community Council directives (86/609/EEC). C57BL6 adult males were deeply anesthetized with ketamine $(100 \mathrm{mg} / \mathrm{kg}$ of body weight; Ketamine 1000; Ceva) and xylazine $(10 \mathrm{mg} / \mathrm{kg}$ of body weight; Rompun 2\%; Centravet) dissolved in $0.9 \%$ sterile saline, and positioned in a stereotaxic frame. Eight animals (male, 3 months-aged mice) per group were injected into the striatum, using appropriate coordinates $(1 \mathrm{~mm}$ anterior to the bregma, $2 \mathrm{~mm}$ lateral, $3 \mathrm{~mm}$ deep from the skull surface) with either eGFP adenovirus or adenovirus expressing human p53 and GFP $\left(10^{7}\right.$ PFU in each hemisphere; Vector Biolabs, Philadelphia, PA, USA). Animals were sacrificed 10 days after injection. Proteins and mRNA were extracted and analyzed as described above. We did Western-blot analysis with the p53 CM1 antibody to show the expression of the injected p53 and GFP.

\section{Statistical analysis}

Statistical analyses were performed with GraphPad Prism software (www.graphpad.com for Windows, CA, USA). No statistical test was used to determine sample size in ex vivo and in vivo studies. No randomization and blindness were used in animal studies. Analysis of two groups of variables was used for samples analyzed by unpaired Student's t-test after having passed a normality test (D'Agostino-Pearse omnibus Normality test) to assure Gaussian distribution of values. All tests are two-sided; the mean was defined as the center value and errors bars correspond to SEM. Analysis of more than two groups of variables (normality test passed) was performed by One-way ANOVA with NewmanKeuls's post-hoc test without any adjustment. The number of samples, replication of experiments and the $\mathrm{P}$ value are provided in figures legends.

Acknowledgements The authors deeply thank Drs. JC. Bourdon, M. Oren, B. Vogelstein, M. Roussel and M. Serrano for providing us either valuable constructs or cellular and animal models. This work was supported by the "Conseil Départemental des Alpes Maritimes", by the Foundation Claude Pompidou and by the University Hospital Federation (FHU) OncoAge. This work has been developed and supported through the LABEX (excellence laboratory, program investment for the future) DISTALZ (Development of Innovative Strategies for a Transdisciplinary approach to ALZheimer's disease). Thomas Goiran is funded by the "Ligue contre le Cancer".

\section{Compliance with ethical standards}

Competing interests The authors declare that they have no competing financial interests.

\section{References}

1. Riley T, Sontag E, Chen P, Levine A. Transcriptional control of human p53-regulated genes. Nat Rev Mol Cell Biol 2008;9 (5):402-12.

2. Maiuri MC, Galluzzi L, Morselli E, Kepp O, Malik SA, Kroemer G. Autophagy regulation by p53. Curr Opin Cell Biol 2010;22 (2): $181-5$.

3. Sui X, Jin L, Huang X, Geng S, He C, Hu X. p53 signaling and autophagy in cancer: a revolutionary strategy could be developed for cancer treatment. Autophagy 2011;7(6):565-71.

4. Tang J, Di J, Cao H, Bai J, Zheng J. p53-mediated autophagic regulation: A prospective strategy for cancer therapy. Cancer Lett 2015;363(2):101-7.

5. Gozuacik D, Kimchi A. Autophagy as a cell death and tumor suppressor mechanism. Oncogene 2004;23(16):2891-906.

6. Crighton D, Wilkinson S, O'Prey J, Syed N, Smith P, Harrison $\mathrm{PR}$, et al. DRAM, a p53-induced modulator of autophagy, is critical for apoptosis. Cell. 2006;126(1):121-34.

7. Tasdemir E, Maiuri MC, Galluzzi L, Vitale I, Djavaheri-Mergny M, D'Amelio M, et al. Regulation of autophagy by cytoplasmic p53. Nat Cell Biol 2008;10(6):676-87.

8. Tasdemir E, Chiara Maiuri M, Morselli E, Criollo A, D'Amelio M, Djavaheri-Mergny M, et al. A dual role of p53 in the control of autophagy. Autophagy 2008;4(6):810-4.

9. da Costa C, Sunyach C, Giaime E, West A, Corti O, Brice A, et al. Transcriptional repression of p53 by parkin and impairment by mutations associated with autosomal recessive juvenile Parkinson's disease. Nat Cell Biol 2009;11(11):1370-5.

10. Zhang C, Lin M, Wu R, Wang X, Yang B, Levine A, et al. Parkin, a p53 target gene, mediates the role of p53 in glucose metabolism and the Warburg effect. Proc Natl Acad Sci U S A. 2011;108 (39):16259-64. 
11. Viotti J, Duplan E, Caillava C, Condat J, Goiran T, Giordano C, et al. Glioma tumor grade correlates with parkin depletion in mutant p53-linked tumors and results from loss of function of p53 transcriptional activity. Oncogene 2014;33 (14):1764-75.

12. Shimura H, Hattori N, Kubo S, Mizuno Y, Asakawa S, Minoshima S, et al. Familial Parkinson disease gene product, parkin, is a ubiquitin-protein ligase. Nat Genet 2000;25(3):302-5.

13. Kondapalli C, Kazlauskaite A, Zhang N, Woodroof HI, Campbell DG, Gourlay R, et al. PINK1 is activated by mitochondrial membrane potential depolarization and stimulates Parkin E3 ligase activity by phosphorylating Serine 65. Open Biol. 2012;2 (5): 120080 .

14. Shiba-Fukushima K, Imai Y, Yoshida S, Ishihama Y, Kanao T, Sato S, et al. PINK1-mediated phosphorylation of the Parkin ubiquitin-like domain primes mitochondrial translocation of Parkin and regulates mitophagy. Sci Rep. 2012;2:1002.

15. Jin SM, Youle RJ. PINK1- and Parkin-mediated mitophagy at a glance. J Cell Sci 2012;125(Pt 4):795-9.

16. Pickrell AM, Youle RJ. The roles of PINK1, parkin, and mitochondrial fidelity in Parkinson's disease. Neuron 2015;85 (2):257-73.

17. Karpinich NO, Tafani M, Rothman RJ, Russo MA, Farber JL. The course of etoposide-induced apoptosis from damage to DNA and p53 activation to mitochodrial release of citochrome C. J Biol Chem 2002;277:16547-52.

18. Komarov PG, Komarova EA, Kondratov RV, Christov-Tselkov $\mathrm{K}$, Coon JS, Chernov MV, et al. A chemical inhibitor of p53 that protects mice from the side effect of cancer therapy. Science 1999;285:1733-7.

19. Narendra DP, Jin SM, Tanaka A, Suen DF, Gautier CA, Shen J, et al. PINK1 is selectively stabilized on impaired mitochondria to activate Parkin. PLoS Biol 2010;8(1):e1000298.

20. Kamijo T, Zindy F, Roussel MF, Quelle DE, Downing JR, Ashmun RA, et al. Tumor suppression at the mouse INK4a locus mediated by the alternative reading frame product p19ARF. Cell. 1997;91(5):649-59.

21. Kudo N, Khochbin S, Nishi K, Kitano K, Yanagida M, Yoshida $\mathrm{M}$, et al. Molecular cloning and cell cycle-dependent expression of mammalian CRM1, a protein involved in nuclear export of proteins. J Biol Chem 1997;272(47):29742-51.

22. Kudo N, Wolff B, Sekimoto T, Schreiner EP, Yoneda Y, Yanagida $\mathrm{M}$, et al. Leptomycin $\mathrm{B}$ inhibition of signal-mediated nuclear export by direct binding to CRM1. Exp Cell Res 1998;242 (2):540-7.

23. O'Keefe $\mathrm{K}, \mathrm{Li} \mathrm{H}$, Zhang Y. Nucleocytoplasmic shuttling of p53 is essential for MDM2-mediated cytoplasmic degradation but not ubiquitination. Mol Cell Biol 2003;23(18):6396-405.

24. Boyd SD, Tsai KY, Jacks T. An intact HDM2 RING-finger domain is required for nuclear exclusion of p53. Nat Cell Biol 2000;2(9):563-8.

25. Goiran T, Duplan E, Chami M, Bourgeois A, El Manaa W, Rouland L, et al. $\beta$-Amyloid Precursor Protein intracellular domain controls mitochondrial function by modulating Phosphatase and Tensin Homolog-induced kinase 1 transcription in cells and in Alzheimer mice models. Biol. Psy. 2017; pii: S0006-3223(17)31515-9. https:// doi.org/10.1016/j.biopsych.2017.04.011. [Epub ahead of print] transcription in cells and in Alzheimer mice models 2017.

26. Lazarou M, Sliter DA, Kane LA, Sarraf SA, Wang C, Burman JL, et al. The ubiquitin kinase PINK1 recruits autophagy receptors to induce mitophagy. Nature 2015;524(7565):309-14.

27. McWilliams TG, Muqit MM. PINK1 and Parkin: emerging themes in mitochondrial homeostasis. Curr Opin Cell Biol 2017;45:83-91.

28. Levine B, Abrams J. p53: The Janus of autophagy? Nat Cell Biol 2008;10(6):637-9.

29. Narendra D, Walker J, Youle R Mitochondrial quality control mediated by PINK1 and Parkin: links to parkinsonism. Cold Spring Harbor perspectives in biology. 2012;4(11).

30. Hoshino A, Mita Y, Okawa Y, Ariyoshi M, Iwai-Kanai E, Ueyama T, et al. Cytosolic p53 inhibits Parkin-mediated mitophagy and promotes mitochondrial dysfunction in the mouse heart. Nat Commun. 2013;4:2308.

31. Clark IE, Dodson MW, Jiang C, Cao JH, Huh JR, Seol JH, et al. Drosophila pink1 is required for mitochondrial function and interacts genetically with parkin. Nature 2006;441(7097):1162-6.

32. Essletzbichler P, Konopka T, Santoro F, Chen D, Gapp BV, Kralovics R, et al. Megabase-scale deletion using CRISPR/Cas9 to generate a fully haploid human cell line. Genome Res 2014;24 (12):2059-65.

33. Mei Y, Zhang Y, Yamamoto K, Xie W, Mak TW, You H. FOXO3a-dependent regulation of Pink1 (Park6) mediates survival signaling in response to cytokine deprivation. Proc Natl Acad Sci U S A. 2009;106(13):5153-8. 